EPJ Web of Conferences 66, 04029 (2014)

DOI: $10.1051 /$ epjconf/ 20146604029

(C) Owned by the authors, published by EDP Sciences, 2014

\title{
Quark spin polarization in high density quark matter
}

\author{
Yasuhiko Tsue ${ }^{1, a}$, João da Providência ${ }^{2}$, Constança Providência² ${ }^{2}$, and Masatoshi Yamamura ${ }^{3}$ \\ ${ }^{1}$ Physics Division, Faculty of Science, Kochi University, Kochi 780-8520, Japan \\ ${ }^{2}$ Departamento de Física, Universidade de Coimbra, 3004-516 Coimbra, Portugal \\ ${ }^{3}$ Department of Pure and Applied Physics, Faculty of Engineering Science, Kansai University, Suita 564- \\ 8680, Japan
}

\begin{abstract}
It is shown that there exists a possibility of spontaneous spin polarization in high density symmetric quark matter under the tensor-type four-point interaction between quarks in the Nambu-Jona-Lasinio model. Further, the spontaneous magnetization is simply estimated by taking into account only the lowest Landau level.
\end{abstract}

\section{Introduction}

One of recent interests about the study of hadronic matter and/or quark-gluon plasma governed by the quantum chromodynamics (QCD) is to understand the phase structure on the plane depicted by the temperature and the baryon chemical potential. In the region of low temperature and high baryon chemical potential in quark matter, rich phase structures are expected such as color superconducting phase including two-flavor color superconducting (2SC) phase, color-flavor locked phase and so on, quarkyonic phase, etc. Especially, by the use of the Nambu-Jona-Lasinio (NJL) model with a pseudovector-type four-point interaction between quarks, a possibility of the spin polarized phase was indicated [1]. However, it was shown that the quark spin polarization disappears at quark mass being zero [2]. Thus, at high baryon density where chiral symmetry is restored and the chiral symmetric phase is realized, the quark spin polarization does not occur because quark masses are zero.

In this paper, another possibility of the existence of spin polarized phase at zero temperature and high baryon density is investigated by using the NJL model with a tensor-type four-point interaction between quarks $[3,4]$. The effective potential for the spin polarization, which corresponds to the expectation value of spin matrix, is considered and the realized phase, namely usual quark phase or the spin polarized phase, is determined by comparing a pressure of one side with another one. The quark spin alignment may lead to the quark ferromagnetization. The spontaneous magnetization is estimated by taking into account the lowest Landau level only.

\section{Effective potential for quark spin polarization}

The NJL type Lagrangian density with a tensor-type four point interaction is introduced to describe the symmetric quark matter as a model Lagrangian density. By considering the high baryon density,

\footnotetext{
ae-mail: tsue@kochi-u.ac.jp
} 
the chiral condensate has no finite value because chiral symmetry is restored. Thus, keeping the mean field approximation in mind, let us start with the following Lagrangian density:

$$
\mathcal{L}=i \bar{\psi} \gamma^{\mu} \partial_{\mu} \psi-\frac{G}{4}\left(\bar{\psi} \gamma^{\mu} \gamma^{\nu} \vec{\tau} \psi\right)\left(\bar{\psi} \gamma_{\mu} \gamma_{\nu} \vec{\tau} \psi\right)
$$

where $\vec{\tau}$ represents the isospin matrix. In the Dirac representation for the Dirac gamma matrices $\gamma^{\mu}$, we get

$$
\gamma^{1} \gamma^{2}=-i \Sigma_{3}=-i\left(\begin{array}{cc}
\sigma_{3} & 0 \\
0 & \sigma_{3}
\end{array}\right)
$$

where $\sigma_{3}$ is the third component of the Pauli spin matrix. Thus, if the vacuum expectation value of $\bar{\psi} \gamma^{1} \gamma^{2} \psi$ has non-zero value, the spin of quarks is aligned along the third axis which leads to the quark spin polarization. Hereafter, it is enough to investigate a possibility of spin alignment along the third axis. The generating functional of the Green function is expressed as

$$
Z \propto \int \mathcal{D} \bar{\psi} \mathcal{D} \psi \exp \left(i \int d^{4} x\left(\bar{\psi} i \gamma^{\mu} \partial_{\mu} \psi+\frac{G}{2}\left(\bar{\psi} \Sigma_{3} \tau_{k} \psi\right)\left(\bar{\psi} \Sigma_{3} \tau_{k} \psi\right)\right)\right) .
$$

Inserting the relation of unity

$$
1=\int \mathcal{D} F_{k} \exp \left(-\frac{i}{2} \int d^{4} x\left(F_{k}+G\left(\bar{\psi} \Sigma_{3} \tau_{k} \psi\right)\right) G^{-1}\left(F_{k}+G\left(\bar{\psi} \Sigma_{3} \tau_{k} \psi\right)\right)\right)
$$

into (3), integrating out $\bar{\psi}$ and $\psi$, introducing the effective action $\Gamma[F]$ as $Z=\exp (i \Gamma[F])$ and defining the effective potential $V[F]$ as $V[F]=-\Gamma[F] / \int d^{4} x$, we obtain

$$
V[F]=\frac{F^{2}}{2 G}-4 N_{c} \int^{F} d F \int \frac{d^{4} p}{i(2 \pi)^{4}}\left[\frac{F-\sqrt{p_{1}^{2}+p_{2}^{2}}}{-p_{0}^{2}+\epsilon_{p}^{(-) 2}}+\frac{F+\sqrt{p_{1}^{2}+p_{2}^{2}}}{-p_{0}^{2}+\epsilon_{p}^{(+) 2}}\right],
$$

where $N_{c}$ represents the number of color and $F_{k} \tau_{k}$ is replaced $F \varepsilon_{\tau}$ with $\varepsilon_{\tau}= \pm 1$ being the eigenvalue of $\tau_{3}$. Here,

$$
\epsilon_{p}^{( \pm)}=\sqrt{\left(F \pm \sqrt{p_{1}^{2}+p_{2}^{2}}\right)^{2}+p_{3}^{2}}
$$

correspond to the single particle energies of quarks. The quark chemical potential $\mu$ is introduced in order to treat the finite density system as $\mathcal{L} \rightarrow \mathcal{L}+\mu \psi^{\dagger} \psi=\mathcal{L}+\bar{\psi} \mu \gamma^{0} \psi$. Thus, we replace $p_{0}$ into $p_{0}+\mu$. Under this replacement, integrating out $p_{0}$ in Eq.(5), we finally obtain the effective potential for quark spin polarization $F$ as

$$
V[F]=\frac{F^{2}}{2 G}+2 N_{c} \int^{F} d F \int_{\frac{d^{3} \boldsymbol{p}}{(2 \pi)^{3}}}\left[\frac{F-\sqrt{p_{1}^{2}+p_{2}^{2}}}{\epsilon_{p}^{(-)}} \theta\left(\mu-\epsilon_{p}^{(-)}\right)+\frac{F+\sqrt{p_{1}^{2}+p_{2}^{2}}}{\epsilon_{p}^{(+)}} \theta\left(\mu-\epsilon_{p}^{(+)}\right)\right],
$$

where $\theta\left(\mu-\epsilon_{p}^{( \pm)}\right)$represents the Heaviside step function.

Next, let us investigate the spontaneous magnetization by considering a response of the external magnetic field $B$. The derivative $\partial_{\mu}$ should be replaced to $D_{\mu}=\partial_{\mu}+i Q A_{\mu}$ where $A_{\mu}=$ $(0, B y / 2,-B x / 2,0)$. After tedious calculation, we obtain the effective potential with external magnetic field $B$, in which the single particle energy $\epsilon_{p}^{( \pm)}$and the momentum integration $\int d p_{1} d p_{2} /(2 \pi)^{2}$ 


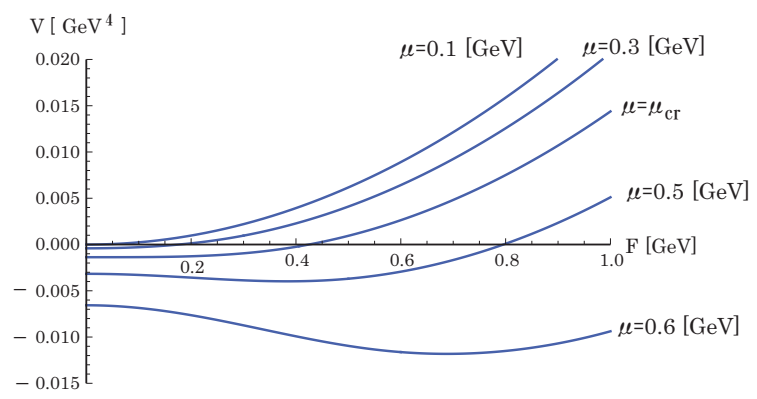

Figure 1. The effective potential is shown as a function of $F$.

are replaced to $E^{( \pm)}=\sqrt{(F \pm \sqrt{2 Q B v})^{2}+p_{3}^{2}}$ and the summation $\sum_{v=v_{\min }(\geq 0)}^{v_{\max }}$. The spontaneous magnetization $\mathcal{M}$ is derived by the thermodynamic relation as

$$
\mathcal{M}=-\left.\frac{\partial V}{\partial B}\right|_{B=0} .
$$

The lowest Landau level here means the level which gives the lowest single particle energy $E^{( \pm)}$. Thus, the lowest Landau level is $v=\left[F^{2} /(2 Q B)\right]$ for $E^{(-)}$and $v=0$ for $E^{(+)}$, where $[\cdots]$ represents the Gauss symbol.

\section{Numerical results and summary}

First, we numerically give the effective potential $V[F]$ in Eq.(7) as a function of quark spin polarization $F$ for various values of chemical potential $\mu$. The model parameter is taken as $G=20 \mathrm{GeV}^{-2}$. Figure 1 shows the effective potential. Up to $\mu=\mu_{\mathrm{cr}}=0.406 \mathrm{GeV}$, the minimum of the effective potential is located at $F=0$. Thus, the normal quark matter phase is realized. However, in the range of $\mu>\mu_{\mathrm{cr}}$, the spin polarization $F$ has non-vanishing value which gives the potential minimum. Figure 2 shows the pressures as a function of (a) the quark chemical potential and (b) the baryon number density divided by the normal nuclear density $\rho_{0}=0.17 \mathrm{fm}^{-3}$. For quark chemical potential $\mu<\mu_{\mathrm{cr}}$, the

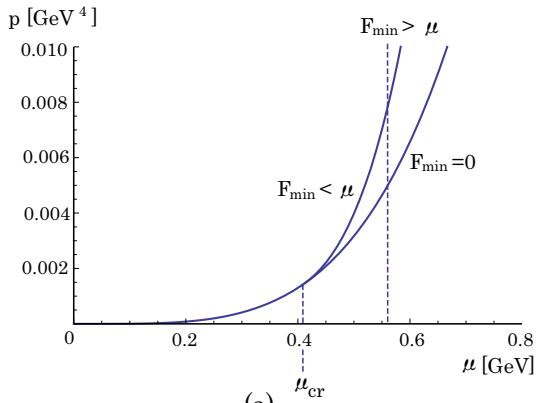

(a)

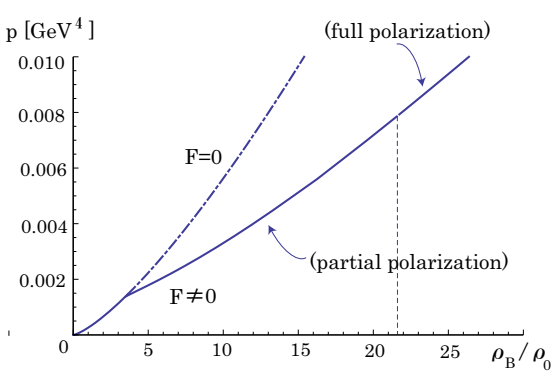

(b)

Figure 2. The pressures as a function of (a) the quark chemical potential and (b) the baryon number density divided by the normal nuclear density $\rho_{0}=0.17 \mathrm{fm}^{-3}$ are shown. 


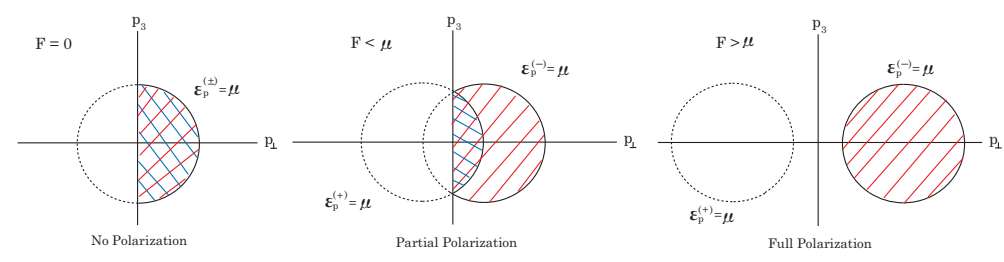

Figure 3. Schematic representations of Fermi surface are given on the plane of $p_{3}$ and $p_{\perp} \equiv \sqrt{p_{1}^{2}+p_{2}^{3}}$.

branch with $F_{\min }=0$ is realized, which leads to the normal quark matter phase. For $\mu>\mu_{\mathrm{cr}}$ the branch with $F_{\text {min }} \neq 0$ is realized, which leads to the spin polarized phase. Here, for $\mu_{\mathrm{cr}}<\mu<0.565$ $\mathrm{GeV}$, the partial spin polarization is realized. For $\mu>0.565 \mathrm{GeV}$, the full spin polarization occurs, in which the Fermi surface is close for the states with the single particle energy $\epsilon_{p}^{(+)}$. Thus, the spin alignment fully occurs. These situations are depicted in Fig.3 schematically. Thus, the quark spin polarization occurs spontaneously for $\mu>\mu_{\mathrm{cr}}$ and the spin polarized phase is realized.

Next, let us estimate the spontaneous magnetization in Eq.(8) numerically by taking into account only the lowest Landau level. Figure 4 (a) shows the spontaneous magnetization $\mathcal{M}$ where the horizontal axis represents the quark chemical potential $\mu$. Also, $\mu_{0} \mathcal{M}$, where $\mu_{0}$ is the magnetic permeability of vacuum, is shown in Fig.4 (b). It is expected that the magnitude of magnetic field induced by the spontaneous magnetization is of order of $10^{16}$ Gauss.

As a conclusion, the quark spin polarized phase is realized at high baryon density against the normal quark matter.

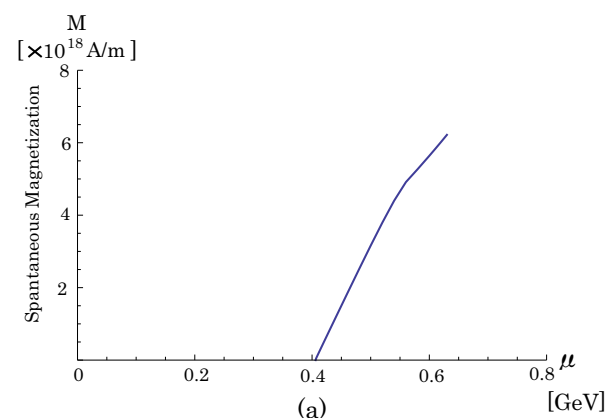

(a)

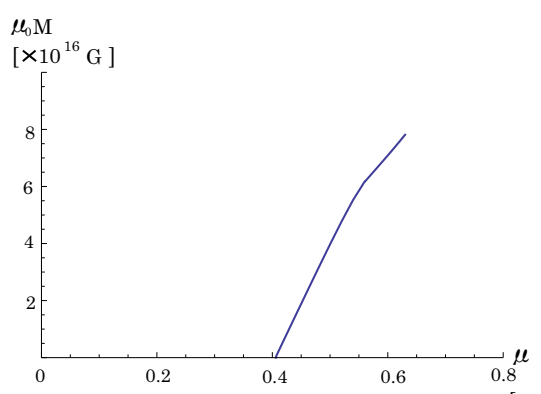

(b)

Figure 4. (a) The spontaneous magnetization and (b) the field strength obtained by multiplying the magnetic permeability of vacuum are depicted as a function of the quark chemical potential.

\section{References}

[1] T. Tatsumi, Phys. Lett. B 489, 280 (2000).

E. Nakano, T. Maruyama and T. Tatsumi, Phys. Rev. D 68, 105001 (2003).

[2] S. Maedan, Prog. Theor. Phys. 118, 729 (2007).

[3] Y. Tsue, J. da Providência, C. Providência and M. Yamamura, Prog. Theor. Phys. 128, 507 (2012).

[4] H. Bohr, P. K. Panda, C. Providência and J. da Providência, Int. J. Mod. Phys. E 22, 1350019 (2013). 